\title{
EL ENLUCIDO EXTERIOR DE YESO EN LAS REHABILITACIONES DE LAS FACHADAS DE PARIS
}

\author{
(REHABILITATION OF GYPSUM PLASTERS IN PARIS)
}

\author{
M. Raymond Pigache \\ Traducción y adaptación: Marina Alvarez Alonso, Arquitecto \\ IETcc/CSIC
}

\section{RESUMEN}

La utilización, fabricación y puesto en obra de morteros de yeso, sobre las fachadas antiguas en el viejo París -técnica puramente manual-, están precisadas desde hace mucho tiempo.

Numerosos enlucidos de mortero de yeso, realizados bajo el reinado de Luis XIV, presentan todavia un excelente comportamiento a pesar de que, como es sabido, el yeso en exteriores es muy sensible a disolverse.

Este estudio ha permitido aclarar cuatro parámetros fundamentales $y$, sobre todo, sus conclusiones han permitido la realización de numerosas rehabilitaciones, tanto en inmuebles de prestigio como en edificaciones modestas:

- El empleo de un mortero cuyos componentes sean más cercanos a los materiales antiguos.

- Una aplicación puramente manual.

- Una proporción establecida a fin de proteger de las aguas de desagüe los enlucidos de mortero de yeso, las cornisas, molduras $y$ etc.

- Muros portantes con las características mecánicas más elevadas que las de los enlucidos.
SUMMARY

The use, the manufacture, the putting into excution of gypsum plaster on old Paris façades -a marely manual technique- are specified since long ago.

A number of gypsum plaster carried out under Louis the 14th reign, have still a good behaviour in spite of, as it is well known, gypsum in the outside is very sensible to dissolution.

This study allows to clarify four fundamental parameters, and its conclusions make it possible to carry out rehabilitations both in prestigeous and in humble buildings:

- The use of a mortar which components will be similar to those old materials;

- A merely handwork.

- An established proportion with a view to protect the gypsum plasters, cornices, moldings, etc. from drainage waters.

- Bearing walls with higher mechanical characteristics than those of platers.

\section{HISTORIA DE LA UTILIZACION DEL YESO EN PARIS}

En el siglo XVIII, las tres cuartas partes de los palacios realizados y la totalidad de los edificios del Estado llano y del Pueblo parisino, fueron realizados en paños de madera tabicados y enlucidos de yeso.

Las construcciones nuevas de la época o las reconstrucciones se hicieron con el $95 \%$ de yeso.

Fue preciso esperar a la revolución industrial del siglo XIX para que este material se abandonara, relativamente, en provecho de materiales modernos (paños metálicos, bloques, etc.).
En efecto, anteriormente el yeso habia sido un material particularmente bien adaptado en las épocas preindustriales.

La leyenda hace remontar el descubrimiento del yeso a un pastor de Montmatre, el cual habiendo utilizado ciertas piedras con el fin de poder encender su hoguera, una lluvia, más súbita que bienechora, convirtio en bloques el conjunto revelando asi las propiedades hidráulicas de la piedra de yeso. Está demostrado que la utilización del yeso fue importada, o al menos desarrollada, por los romanos. Ellos, muy respetuosos de la tradición, asumieron las afirmaciones de Vitrubio: "era un salvaje todo hombre que no habitaba en una morada estable» pero, siendo 


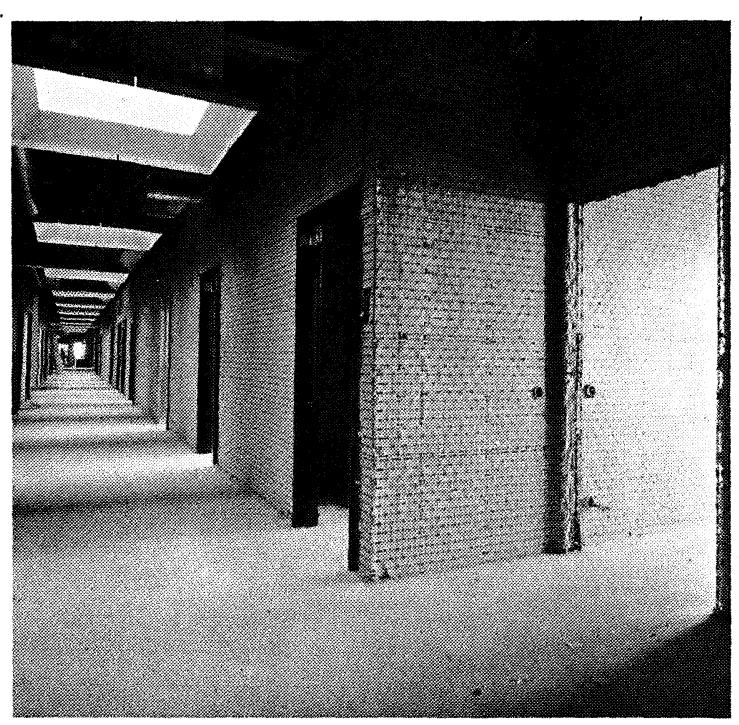

Uso de técnicas muy actuales en el interior.

igualmente economistas (la piedra labrada era rara o dificilmente explotable), prefirieron utilizar el yeso.

Toda una civilización del yeso iba a nacer y prosperar en Paris, lo que hizo escribir a Raoul Brotaye, abogado del gran Consejo del siglo XVI: «el yeso es tan útil y tan empleado en la construcción de nuestras casas que, por la resplandeciente blancura con la que las reviste, hace de Paris una ciudad del yeso".

Más que histórica y anecdótica, la utilización del yeso en Paris ha sido particularmente ventajosa en las eras preindustriales. La piedra de yeso estaba disponible en una cercania inmediata a su lugar de empleo, lo que permite decir "hay más de Montmatre en Paris, que de París en Montmatre».

Este material se encuentra a muy poca profundidad y permite una extracción fácil.

Su transformación en aglomerante, a una temperatura poco elevada obtenida fácilmente en instalaciones simples, y un molido grosero, completado por un tamizado artesanal, permite debilitar costes de explotación.

Asi, si generalmente se le hace remontar a la época de Luis XIV, éste impresionado por el incendio de Londres en 1666 y ordenando el 18 agosto de 1667 recubrir los paños de madera por yeso, se constata en la época la reconstrucción de edificios a los que no se les hubiera tenido en cuenta para esto (construcción anterior adaptada, ausencia de antemano de ripias de castaño, etc.: el Estado no habia, como a veces ocurre, homologado y oficializado las prácticas corrientes.

Superficies teniendo una perennidad de varios siglos, a pesar de que se conoce el mal compor- tamiento de los yesos modernos en el exterior, han conducido a numerosos investigadores a analizar estos enlucidos.

\section{COMPOSICION DE LOS MORTEROS DE YESO ANTIGUOS}

Es importante constatar que los análisis efectuados presentan una composición de los morteros de yeso de una notable regularidad.

Numerosos son los autores que encuentran una fórmula cercana a:

1. Volumen de cal.

2. Volúmenes de arena.

3. Volúmenes de yeso.

En su reemplazamiento en el contexto técnico, que ha presidido la fabricación del yeso, se constata que el horno o sus derivados se superponen cronológicamente a la realización de los morteros de yeso.

\subsection{Los yesos de hornos}

La descripción del químico Malaguitti en 1866 puede ser aplicada a la fabricación del yeso desde la antigüedad.

El quimico Malaguitti describe asi, en 1866, la fabricación del yeso:

"Se construye por medio de gruesos bloques de piedra de yeso pequeñas bóvedas poco altas, que se les carga con fragmentos de este mineral, situando gradualmente los más grandes en la base y los menos voluminosos en lo alto.

Se hace quemando madera seca bajo las bóvedas: cuanto más lenta y regular sea la cocción, mejores son los productos. La operación dura un promedio de 10 horas: cuando se acaba, se ciegan las aberturas con bloques y se recubre la base con yeso en polvo.

Es evidente que la masa no puede estar igualmente calcinada en todas sus partes. La porción que está más cerca del fuego está bastante calcinada para fraguar con el agua, es pues inerte; la que está más alejada, está todavia demasiado hidratada, pero no inerte: entre estos dos extremos existe lo que está en el punto requerido: todo unido dará un yeso de excelente calidad. En efecto, el que ha sido demasiado calcinado jugará el papel de un cuerpo extraño, desde que está reconocido que un buen yeso para construcción no debe ser puro. En lugar de operar de esta manera, se puede cocer el yeso con cuidado y entonces añadir enseguida materias extrañas sin acción. 
Cuando el yeso no está bastante calcinado, es árido y seco: demasiado calcinado no tiene "amour» lo que quiere decir en el lenguaje de los obreros de Paris, que no es bastante graso, calcinado a propósito, es casi untuoso al tocarlo, y se adhiere a los dedos".

Las observaciones descritas por Malaguitti, pueden desde ahora en adelante permitirnos reconstruir la composición de un yeso de esta época. Contiene todas las fases de deshidratación del yeso: crudo, semihidratado $\beta$, anhidritas no solubles, sobrecalcinado y cal.

Ahora bien, hacia los años 1930 las técnicas de fabricación del yeso sufren profundas mutaciones. Las cualidades o defectos (según) que eran mantenidas durante siglos desaparecen en provecho de un yeso más fino, más homogéneo: esencialmente el semihidratado. Se constata muy a menudo que los morteros de yeso, que han sido realizados con este material, tienen un comportamiento desastroso: fisuraciones, desprendimientos, pulverulencias.

De la multiplicidad de las fases mineralógicas, que tienen un buen comportamiento, $s \in$ ha pasado a un monocomponente inestable.

Esta constatación ha conducido a investigar, entre los yesos especiales, aquéllos que tienen caracteristicas físicas comparables: son los yesos en conformidad con la Norma PGC1, yeso grueso de construcción pero igualmente de caracteristicas mineralógicas comparables; es decir, un material que contiene además de semihidratado (cocción entre 110 y $160^{\circ} \mathrm{C}$ ) anhidrita y, más particularmente, la anhidrita llamada de otra forma yeso hidráulico obtenido en los hornos a una cocción sobre $500^{\circ} \mathrm{C}$.

Actualmente, es fácil obtener yeso PGC1 destinado a la fabricación de ladrillos de yeso, que es únicamente un semihidratado muy soluble. Su utilización antes arriesgada conduce a comportamientos desastrosos comparables a la de los yesos finos tipo PFC, levantando numerosas interrogaciones de los prescriptores y los usuarios.

No existe actualmente más que un yeso granulométrica y mineralógicamente comparable.

Los yesos antiguos contenian una proporción de inquemados y de yeso "cocido hasta la muerte" (entre 600 y $900 \circ \mathrm{C}$ incapaz de fraguar) que, como señala Malaguitti, servia de cargas inertes. Por otra parte, se le añadia frecuentemente arena.

\subsection{Las arenas}

Mientras que los semihidratos cristalizan bajo forma de hojas casi bidimensionales, la anhidrita

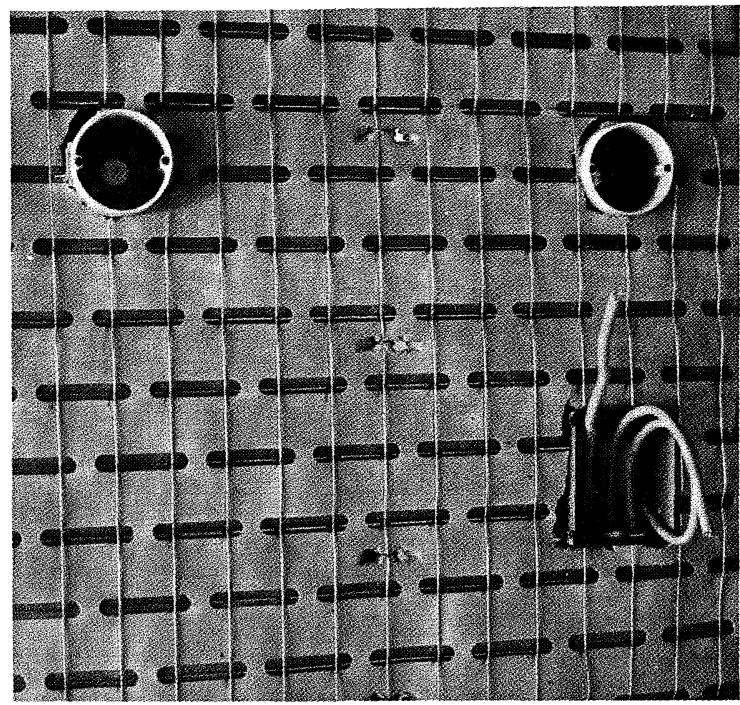

Rehabilitacion de paramentos interiores con sistemas avanzados.

da una red más tridimensional. El añadido de una arena relativamente fina favorece esta cristalización tridimensional dando "cuerpo" al enlucido.

Estas arenas deben ser apropiadas; la presencia de arcillas expansivas como las montmorillonitas tienen el riesgo de que con eventuales humedades deterioran las redes de cristalización.

De la misma forma, una granulometría demasiado grande crea diaclasas y así los riesgos de ruptura favorecen la infiltración y la permanencia de las aguas atmosféricas. Una protección eficaz contra estas aguas liquidas puede estar asegurada por la adición de cal.

\subsection{Las cales}

El mundo de las cales es muy amplio: de la cal aérea (llamada así porque endurece por absorción del gas carbónico del aire), a las cales eminentemente hidráulicas (fraguadas en agua, contienen prácticamente todos los minerales de los cementos Portland), existen todos los intermedios.

Ahora bien, las propiedades hidráulicas de los arracimados (granzones muy duros obtenidos a través de la calcinación de calcáreos impuros) han sido revelados en el curso del siglo XIX; quizá porque los fabricantes habian molido el conjunto del resultado de la cocción para evitar un hilado oneroso.

Se puede pues admitir que en los morteros de yeso, tales como los que se habian realizado antes, no se utilizaba más que cal aérea.

Por otra parte, los procesos de fabricación que obligaban a dejar la cal viva apagarse, durante varios meses en fosas, habrian de todas formas neutralizado los elementos hidráulicos. 


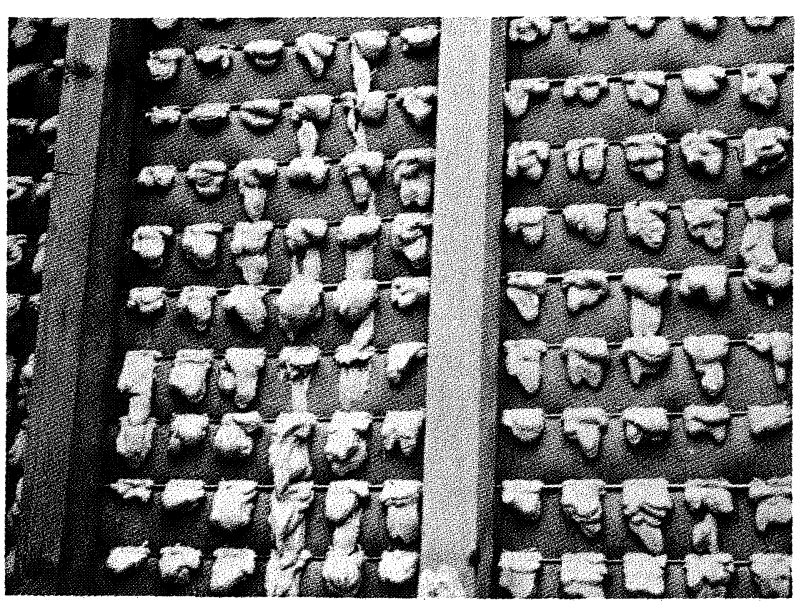

La aplicación de paneles interiores de doblado se realiza con yeso.

Asimismo se conocen importantes desórdenes ocasionados por la Sal de Candlot (ettringita) que aparece frecuentemente cuando se aplica yeso sobre el cemento; es poco razonable introducir los compuestos anhidros del cemento Portland en el yeso.

Los ensayos realizados por falta de información sobre las variedades de cal han conducido, debido al empleo de cales hidráulicas en estos últimos años, a pulverulencias e hinchamientos cercanos a la ruina total del enlucido.

Es pues imperativo no utilizar más que una cal aérea perfectamente apagada en proporciones adaptadas. En efecto, estando señalada la dimensión del cristal de calcita resultante de la carbonatación del hidróxido:

- Romboedro de hidróxido de calcio $C=5.026 \mathrm{~A}$.

- Romboedro de calcita parámetro $C=6.36 \mathrm{~A}$.

- Hay un aumento de volumen de cristal por integración de carbono en la malla cristalina; es verosimil que una red de cristal, poco dura, juegue el papel de tapaporos en el seno de cristalita de yeso y que una cantidad demasiado grande entrañaria una verdadera estructura cristalina que, por falta de espacio, haria reventar la trama de agujas de yeso.

\section{PUESTA EN OBRA}

Refiriéndose siempre a las técnicas antiguas, parece que la puesta en obra era muy simple. La rehabilitación de las antiguas fachadas de mortero de yeso, el redescubrimiento de una mano de obra especializada -que muchos consideraban como desaparecida-, ha permitido trasladarse en el tiempo. En efecto, la técnica utilizada actualmente parece no diferir de la del siglo XVIII más que por el andamiaje metálico y las reglas de composición metálicas. Ahora bien, se percibe rápidamente que esto es erróneo: Las compo- siciones en madera son mucho más fáciles de emplear (en el momento la madera hincha ligeramente y después se seca; es muy fácil de levantar sin riesgo de fracturas).

El material consiste pues:

- andamiaje,

- piezas en madera o materia plástica,

- mezclador,

- paletas de acero o cobre (esta última es más corriente en la decoración de los despachos de arquitectura que en las obras),

- plantilla.

\subsection{Puesta en obra}

El mortero de yeso debe ser amasado relativamente denso $(Y / a=1,47)$.

Su período de trabajabilidad de 10 a $15 \mathrm{~mm}$ no permite más que mezclas manuales saco por saco.

La colocación se puede efectuar:

Una capa: después de un primer enlucido, el mortero de yeso es aplicado sobre el soporte, tendido y compacto con la llana y después cortado y raspado.

Enlucido en dos capas: cuando esta primera capa ha alcanzado una dureza suficiente, se corta. La 2.a capa será aplicada enseguida, el grado de mezcla no debe ser inferior al de la 1. a capa.

Estos dos pasos serán aplicados en un lapso de tiempo suficientemente próximo para no constituir más que una sola capa: estando la 1.a más compactada que la 2.a.

\subsection{El modo de colocación}

Esta noción de sujeción que en los siglos precedentes formaba parte de las reglas del arte ha sido poco a poco perdida en beneficio de la proyección mecánica. Ahora bien, en 1984, M. Toussaint anota en su "Nuevo manual completo del albañil»: Desde hace algunos años se ha recurrido a un medio más expeditivo, el de proyectar el enlucido a escoba como el tendido y con el yeso mezclado claro, absolutamente en la misma forma: este uso es tolerado sin razón porque primeramente el yeso es lanzado a menor consistencia que el primero y por lo tanto es menos adherente que con la paleta; también a menudo forma ampollas y huecos que se desgarran por trozos después de algunos meses.

Desde esta época, diversos toques de atención eran dirigidos a los usuarios de métodos de proyección que empezaban a nacer. 
Pero contrariamente a las técnicas modernas, regidas por los DTV en las cuales el soporte está esencialmente descrito, los morteros de yeso y cal antiguos tenian en cuenta el soporte y la proporción.

\subsection{El sistema constructivo}

\subsubsection{La protección de los enlucidos}

El yeso es un material soluble en el agua, y cualesquiera que sean las mejoras, debidas a composiciones enriquecidas por la cal, por la anhidrita, esta eventualidad no se puede descartar jamás. También arquitectos y enlucidores han confrontado siempre su experiencia. Previendo entablamentos, cornisas, molduras, techos salientes, etc., el arquitecto, logrando la estética de las fachadas, aseguraba la protección de los enlucidos contra las aguas de desagüe.

Todas estas molduras permitian la fijación de baberos y láminas protectoras de cinc, sin olvidar la gota de agua bajo las molduras que ha tenido desgraciadamente una desdichada tendencia a degenerar en el curso del tiempo hasta hacerlos desaparecer en ciertas construcciones modernas.

\subsubsection{Los materiales de construcción}

Herederos de una larga tradicción, los soportes están esencialmente constituidos de cimientos de piedra dura formando barrera de capilaridad, y de paños de madera con un relleno heteroclito de paredes macizas de bloques frecuentemente colmados con yeso o con cal.

Estos dos tipos de paredes tenian dos características: Una masa importante y una gran flexibilidad. $Y$ es notable que estas construcciones presentan frecuentemente deformaciones importantes: desplome y contradesplome, combas y desplomes por los cuales el enlucido de yeso y cal ha fluido para adaptarse a las deformaciones.
De esta propiedad de deformabilidad no interesa más que los movimientos lentos de grandes amplitudes: para los cizallamientos puntuales, tales como los asientos diferenciales de entrepaños, el comportamiento es tan deplorable como el de un mortero de cemento (pero los enlucidos no tienen, de cualquier manera, vocación de mantenimiento de estructuras).

\section{CONCLUSIONES}

Parece, por el análisis de rehabilitaciones de fachadas antiguas de Paris, que el mortero de yeso y cal, heredero de una larga tradición, está constituido por materiales económicos de caracteristicas bien definidas.

Es muy probable que sean los costes de fabricación débiles.y las técnicas relativamente simples los que han permitido a estos dos materiales ser los aglomerantes más antiguos, habiendo alcanzado un estado de desarrollo preindustrial.

Esto está de hecho igualmente unido a la proximidad y a la facilidad de explotación de las materias primas. En contrapartida, esta mezcla de un empleo delicado necesita una mano de obra perfectamente cualificada y sobre todo fuertemente motivada, capaz de compensar la simplicidad del utillaje por una gran habilidad.

De igual forma no permite rendimientos importantes compatibles con las normas de rentabilidad occidentales.

Forma parte de un mercado tan tradicional que los documentos técnicos codificados, constituidos por la colección de los métodos tradicionales, se han olvidado.

Este tipo de revestimiento es indisociable de la arquitectura; debe estar perfectamente integrado como un parámetro tan fundamental como el cálculo de las estructuras en la concepción de los proyectos. 